\title{
CD19 Positive
}

National Cancer Institute

\section{Source}

National Cancer Institute. CD19 Positive. NCI Thesaurus. Code C129255.

Indicates that CD19 expression has been detected in a sample. 